Specific symptoms of the General Health Questionnaire (GHQ) in predicting persistence of psychological distress : Data from two prospective cohort studies

\author{
Jokela, Markus
}

$2021-11$

Jokela , M , Velázquez, R G , Komulainen , K, Savelieva , K, Airaksinen , J \& Gluschkoff , K 2021 , ' Specific symptoms of the General Health Questionnaire (GHQ) in predicting persistence of psychological distress : Data from two prospective cohort studies ', Journal of Psychiatric Research , vol. 143 , pp. 550-555 . https://doi.org/10.1016/j.jpsychires.2020.11.026

http://hdl.handle.net/10138/336292

https://doi.org/10.1016/j.jpsychires.2020.11.026

unspecified

submittedVersion

Downloaded from Helda, University of Helsinki institutional repository.

This is an electronic reprint of the original article.

This reprint may differ from the original in pagination and typographic detail.

Please cite the original version. 


\title{
Specific symptoms of the General Health Questionnaire (GHQ) in predicting persistence of psychological distress: Data from two prospective cohort studies
}

\author{
Markus Jokela, ${ }^{1}$ Regina García Velázquez, ${ }^{1}$ Kaisla Komulainen, ${ }^{1}$ Kateryna Savelieva, ${ }^{1}$ \\ Jaakko Airaksinen, ${ }^{2}$ Kia Gluschkoff ${ }^{1,3}$ \\ 1 Department of Psychology and Logopedics \\ 2 Institute of Criminology and Legal Policy \\ 3 Finnish Institute for Health and Welfare
}

Manuscript statistics: 170 words in abstract, 2259 words in text, 1 table, 2 figures, 4 supplementary figures

Corresponding author: Dr. Markus Jokela, Department of Psychology and Logopedics, University of Helsinki, 00014 University of Helsinki, Finland. E-mail:

markus.jokela@helsinki.fi

Funding: The research was financially supported by the Academy of Finland (grant: 311578).

Acknowledgements: Understanding Society is an initiative funded by the Economic and Social Research Council and various Government Departments, with scientific leadership by the Institute for Social and Economic Research, University of Essex, and survey delivery by NatCen Social Research and Kantar Public. The research data are distributed by the UK Data Service. Neither the original collectors of the data nor the distributors of the data bear any responsibility for the analyses or interpretations presented here. Data references: University of Essex. Institute for Social and Economic Research, NatCen Social Research and Kantar Public, [producers]: Understanding Society: Waves 1-6, 2009-2015 [computer file]. 8th Edition. Colchester, Essex: UK Data Service [distributor], November 2016. SN: 6614. University of Essex. Institute for Social and Economic Research. (2010). British Household Panel Survey: Waves 1-18, 1991-2009. [data collection]. 7th Edition. UK Data Service. SN: 5151, http://doi.org/10.5255/UKDA-SN-5151-1

Author contributions: Markus Jokela: Formal analysis, Funding acquisition, Writing; All authors: Conceptualization, Methodology, Validation, Writing - review \& editing.

Competing interests: None of the authors have any competing interests. 
Specific symptoms of the General Health Questionnaire (GHQ) in predicting persistence of psychological distress: Data from two prospective cohort studies

Manuscript statistics: 170 words in abstract, 2259 words in text, 1 table, 2 figures, 4 supplementary figures 


\section{Highlights}

- Persistence of psychological distress may be related to the constellation of specific symptoms

- Sense of worthlessness, loss of self-confidence, loss of sleep over worry, and feelings of strain were most systematically related to persistent distress

- General happiness, feelings of unhappiness or depressed mood, and enjoyment of activities showed no independent associations with persistence of distress

- Symptom-specific assessment of psychological distress may be helpful in identifying high versus low risk of distress persistence over time 


\begin{abstract}
Persistent psychological distress is more harmful than transient psychological distress, but little is known about the development of persistent distress. We examined whether some specific symptoms of the 12-item General Health Questionnaire (GHQ-12) were more important than others in predicting the persistence of psychological distress over a 3-year follow-up period among individuals who had at least moderate psychological distress at baseline (GHQ $\geq 3$ ). Participants were from the UK Household Longitudinal Study (UKHLS; n=6,430) and British Household Panel Survey (BHPS; n=5,954). More persistent distress had dose-response associations with symptoms of sense of worthlessness, loss of self-confidence, loss of sleep over worry, and feelings of strain. General happiness, feelings of unhappiness or depressed mood, and enjoyment of activities showed no dose-response associations. Symptoms of social functioning (capability of making decisions, concentration problems, feelings of usefulness, ability to face problems) showed some but not consistent dose-response associations. These results suggest that feelings of worthlessness, loss of self-confidence, loss of sleep over worry, and strain may be particularly important markers for persistent psychological distress.
\end{abstract}

Keywords: Psychological distress; Persistence; Longitudinal; Cohort study; General Health Questionnaire; Complicated depression 
Mental health problems in the general population are often assessed with measures of non-specific psychological distress that cover symptoms of anxiety, depression, stress, and somatic complaints (1). Such symptoms are common in the population, with nearly $20 \%$ of people reporting at least moderately severe psychological distress. For some individuals, psychological distress is only temporary and wanes within months. For others, psychological distress can become chronic and last for several years. Compared to acute distress, chronic psychological distress can be expected to have more adverse consequences than short-term acute distress. Therefore, it is crucial to better understand the development of chronic psychological distress.

The persistence of psychological distress may partly depend on the specific symptoms of distress $(5,6)$. While the specific symptoms of distress are often considered merely as interchangeable indicators of overall psychological distress, there is evidence to suggest that some of the specific symptoms may be differently related to risk factors and outcomes of distress (7-11). Regarding persistence, studies of major depressive disorder have suggested a difference between “complicated” and “uncomplicated” manifestations of depression (6,12,13). Complicated depression is characterized by sense of worthlessness, suicidal ideation, psychotic ideation, psychomotor retardation, depressive episodes lasting more than six months, and marked functional impairment. Uncomplicated depression, by contrast, is suggested to represent a normal distress response to stressful life circumstances and to be characterized by sadness, lessened appetite, insomnia, concentration difficulties, and fatigue. Compared to uncomplicated depression, complicated depression has been associated with longer episodes and increased risk of persistence of depression $(5,6,12,13)$. Similar findings have been reported for depressive symptoms that do not reach the clinical thresholds of diagnosis (12), which implies that the complicated symptoms may be particularly important already in the pre-clinical stage.

The purpose of the present study was to examine whether specific symptoms of the widely used 12-item General Health Questionnaire (GHQ-12; (14)) are differently related to the persistence of overall psychological distress over time. Based on the difference between complicated vs uncomplicated depression (5), we hypothesized that worthlessness would predict more chronic psychological distress while the symptoms related to sadness (depressed mood and general happiness), loss of sleep over worry, and concentration problems would be less important predictors of chronicity. We did not have specific hypotheses about other symptoms of the GHQ.

\section{Materials and methods}

Data were from the UK Household Longitudinal Study (UKHLS; (15)) and the British Household Panel Survey (BHPS; (16)). The BHPS was a longitudinal survey of a nationally 
representative sample of over 5000 British households with annual follow-ups. The original cohort included 10,264 individuals aged 16-97 at baseline in 1991, and was based on a clustered, stratified sample of addresses throughout Great Britain south of the Caledonian Canal (excluding North of Scotland and Northern Ireland). New participants were included in the sample over the years if they were born to original sample member, if they had moved into a household in the original sample, or if a member of the original sample moved into a new household with one or more new people. In addition, the sample was enriched with additional recruitment of participants at waves 9 and 11, from Scotland and Wales, and from Northern Ireland, respectively, extending the sample to cover the whole UK. The $18^{\text {th }}$ and final follow-up of the BHPS was carried out in 2008-2009.

After 2008-2009, the BHPS became merged with the larger UKHLS, also known as the Understanding Society study (http://www.understandingsociety.org.uk/). The original participants for the UKHLS were from approximately 40,000 household in the United Kingdom, including participants from the BHPS, interviewed in 2009-2011. The overall purpose of Understanding Society is to provide high quality longitudinal data about subjects such as health, work, education, income, family, and social life to help understand the long-term effects of social and economic change, as well as policy interventions designed to impact upon the general well-being of the UK population. The $8^{\text {th }}$ and most recent follow-up for which data were available for the present analysis was carried out in 2016-2018.

The 12-item General Health Questionnaire (GHQ-12; (14)) asks whether the respondent has recently (a) been able to concentrate on whatever you're doing, (b) lost much sleep over worry, (c) felt that you were playing a useful part in things, (d) felt capable of making decisions about things, (e) felt constantly under strain, (f) felt you couldn’t overcome your difficulties, (g) been able to enjoy your normal day-to-day activities, (h) been able to face up to problems, (i) been feeling unhappy or depressed, $(j)$ been losing confidence in yourself, $(k)$ been thinking of yourself as a worthless person, and (l) been feeling reasonably happy, all things considered. For the negatively worded items the response scale was: $1=$ Not at all, 2=No more than usual, 3=More than usual, 4=Much more than usual. For the positively worded items the response scale was: 1=More than usual, 2=Same as usual, 3=Less than usual, 4=Much less than usual. Following the common scoring method of GHQ items, responses of 3=More than usual and 4=Much more than usual (for negatively worded items) and 3=Less so than usual and 4=Much less than usual (for positively worded items) indicated the presence of the symptom. A sum score of $\geq 3$ was used as a cut-off indicating at least moderate psychological distress (GHQ caseness). Covariates included sex, age, and ethnic background ( $0=$ White British, $1=$ Other). As a complement of the conventional GHQ scoring, we also fitted the models using the alternative “Chronic GHQ” scoring method (CGHQ; 
$(17,18))$ in which six of the positively worded items are scored as in the conventional scoring method $(1=0,2=0,3=1,4=1)$ while the negatively worded items are scored so that only the complete absence of the symptom is scored as zero $(1=0,2=1,3=1,4=1)$. We used the same cut-off value of $\geq 3$ for the CGHQ in calculating the number of GHQ caseness over the three-year followup periods.

\section{Statistical analysis}

Given that our analysis focused on the persistence (rather than incidence) of psychological distress, the analytic sample of each data cycle consisted of individuals who had a GHQ sum score of $\geq 3$ at baseline. We examined how the specific GHQ symptoms at data-cycle baseline predicted the number of times the participant was a GHQ case in the next three study waves. The follow-up was limited to 3 years because in the total sample fewer than $5 \%$ of the participants would have been a GHQ case for four times in a 4-year follow-up period.

Based on these four-year cycles (baseline plus three follow-up years), multiple personobservations for the same individuals were included in the analyses by stacking the data into nonoverlapping follow-up cycles in which GHQ symptoms at wave 1 were used to predict GHQ caseness in waves 2, 3, and 4; GHQ symptoms at wave 5 were used to predict GHQ caseness in waves 6, 7, and 8 in both cohorts. Additionally in the BHPS, GHQ symptoms at wave 9 predicted GHQ caseness in waves 10,11, and 12; and GHQ symptoms at wave 13 predicted GHQ caseness in waves 14, 15, and 16 (BHPS waves 17 and 18 were not included in the analysis). This provided a maximum of two person-observations from the same participants in UKHLS (8 waves in total) and a maximum of 4 person-observations in the BHPS (16 waves in total). When used as predictors, the individual items were coded on the original 4-point scale (instead of dichotomous coding).

The associations of specific symptoms with the persistence of GHQ caseness was assessed with multinomial logistic regression with all the GHQ symptoms and covariates as exposure variables and the four-year cycle GHQ caseness sum score as the outcome. We assessed the linear trends of the symptoms to examine whether the specific symptoms followed a dose-response association in predicting the number of times the person was a GHQ case during the three-year follow-up: the stronger the dose-response association, the more important the symptom was considered to be for persistent psychological distress. Robust estimator with the participant as the clustering factor was used to estimate standard errors to account for the non-independence of the person-observations in the dataset. To account for missing data, we used a $n=1$ stochastic imputation to impute missing values of individual symptoms and GHQ caseness sum score for each data cycle for individuals who had data for at least one measurement time of GHQ caseness for the 
data cycle in question. For example, missing values for data cycle spanning from wave 5 to 8 were not imputed for individuals who did not have any assessments of GHQ caseness in waves 6, 7, and 8.

\section{Results}

Descriptive statistics of the participants with GHQ score of $\geq 3$ at least in one of the baseline waves of 1, 5, 9, and 13 are shown in Table 1 ( $\mathrm{n}=6430$ in UKHLS; $\mathrm{n}=5954$ in BHPS). Figure 1 shows the main results for UKHLS and Figure 2 shows the corresponding results for BHPS. The most consistent evidence for dose-response associations observed in both cohorts were for worthlessness, loss of self-confidence, loss of sleep over worry, and feelings of strain. Symptoms of enjoyment of daily activities, feeling unhappy or depressed, and general happiness showed no evidence of dose-response association in predicting the persistence of GHQ caseness in either cohort. Symptoms related to social functioning (ability to concentrate, capability to overcome difficulties, decision making, and ability to face problems) showed some but not consistent doseresponse associations. Supplementary analyses with imputed data led largely to the same conclusions (Supplementary Figures 1 and 2; n=12,817 person-observations of 10.816 participants in UKHLS; $n=19,879$ person-observations of 9,766 participants in BHPS), except for a strengthening association for being depressed/unhappy in both cohorts. Using the CGHQ coding when calculating the GHQ sum score led to strengthening of some of the dose-response associations in the UKHLS (Supplementary Figure 3) but to attenuation of most of the associations in the BHPS (e.g., feelings of worthlessness and loss of sleep over worry retaining their dose-response associations; Supplementary Figure 4).

\section{Discussion}

We hypothesized that some symptoms of psychological distress might be more important than others in predicting the persistence of distress in the general population. Our results showed that sense of worthlessness, loss of self-confidence, loss of sleep over worry, and feelings of strain were the most consistent symptoms in predicting increasingly persistent distress over the following three years. By contrast, increasingly persistent distress was not independently predicted by general happiness, being unhappy or depressed, loss of enjoyment, capability of making decisions, concentration problems, feelings of usefulness, and ability to overcome difficulties or face problems. The results support the hypothesis that some symptoms are more important than others for persistence of psychological distress. 
Before interpreting the findings, it is important to consider some study limitations. The selfreported format of the GHQ may introduce imprecision into the assessment of specific symptoms, as people may interpret the items somewhat differently. The overlap between specific symptoms might increase if the respondents cannot sufficiently differentiate between the psychological content of the items. Second, the GHQ asks whether the respondent has experienced the symptoms "more than usual”, which may bias the responses of individuals who tend to be highly distressed in general, for example, due to their personality dispositions (17). On the other hand, the emphasis on unusual levels of distress may be useful in specifically measuring the psychological distress that goes over and above the person's habitual level of negative emotions (19). Third, the GHQ-12 covers only some of the possible symptoms related to depression, anxiety, and somatic complaints, and it does not include some relevant symptoms such as suicidal ideation or changes in appetite (20). Also, the only symptom related to sleep problems asks whether the person has lost sleep over worry, which makes this item more of a symptom of anxiety than insomnia (21). Therefore, the role of broader range of symptoms in persistent distress needs to be examined with other measures of psychological distress besides the GHQ-12.

Our findings partly align with the suggested difference between complicated and uncomplicated depression $(5,22)$. Sense of worthlessness, suicidal ideation, psychotic ideation, and psychomotor retardation have been suggested to indicate more pathological and recurrent forms of depression (13). We observed that the symptoms of worthlessness and loss of self-confidence were most strongly associated with persistent distress. By contrast, symptoms of sadness, appetite, insomnia, concentration difficulties, and fatigue are suggested to represent "uncomplicated” responses to stressful circumstances (22). We observed that the core symptoms of major depression (depressed mood and loss of enjoyment) and general unhappiness were not independently associated with increasing risk of chronic distress. Within the confines of symptoms covered by the GHQ-12, these symptom-specific associations support a difference between "complicated” and “uncomplicated” psychological distress. The symptoms related to social functioning (feeling useful, capable of making decisions, able to overcome difficulties, and face problems) showed some but not consistent evidence for dose-response associations. Limitations in social functioning may reflect both the adverse effects of psychopathology as well as difficulties in dealing with difficult and stressful life circumstances.

In conclusion, our findings suggest that feelings of low self-worth, worry, and strain may be particularly important symptoms in characterizing more chronic forms of psychological distress. These symptoms may thus be indicators of more complex psychological distress as compared to more normal stress people encounter from time to time in their daily lives. If these findings are 
replicated with other measures of psychological distress, the symptom-specific associations might have implications for more accurate screening for persistent and complex psychological distress in the population, and the most effective treatment protocols that target the symptoms most strongly associated with persistent distress. 
Table 1. Descriptive statistics for individuals with $G H Q$ score $\geq 3$ at data-cycle baseline

\begin{tabular}{lcc}
\hline Variable & UKHLS & BHPS \\
\hline Age $^{*}$ & $46.3(15.6)$ & $44.5(17.1)$ \\
Females & $64.1(4585)$ & $62.6(5259)$ \\
Ethnic minority & $16.9(1206)$ & $4.5(381)$ \\
GHQ caseness score & & \\
$\quad$ Zero & $30.73(2,198)$ & $23.58(1,982)$ \\
One & $26.94(1,927)$ & $25.44(2,138)$ \\
Two & $22.24(1,591)$ & $25.57(2,149)$ \\
$\quad$ Three & $20.09(1,437)$ & $25.41(2,136)$ \\
\hline $\mathrm{n}$ (persons) & 6430 & 5954 \\
$\mathrm{n}$ (person-observations) & 7153 & 8405
\end{tabular}

Note: Values are percentages (and numbers) of participants unless otherwise noted. *

Values are means (and standard deviations). UKHLS = UK Household Longitudinal

Study, BHPS = British Household Panel Survey 


\section{Figure captions}

Figure 1. Associations of GHQ-12 items in the UKHLS in predicting the number of times the participant was a GHQ case in the following 3 study waves (One, Two, Three; the reference group being zero). Values are natural logarithms of relative risks derived from a multinomial logistic regression. The Trend column gives the linear dose-response association for the symptom across the numbers of GHQ caseness. The GHQ items are all scored on a 4-point response scale, with higher values indicating higher psychological distress. $\mathrm{N}=7,153$ person-observations of 6,430 participants with GHQ $\geq 3$ at data-cycle baseline.

Figure 2. Associations of GHQ-12 items in the BHPS in predicting the number of times the participant was a GHQ case in the following 3 study (One, Two, Three; the reference group being zero). Values are natural logarithms of relative risks derived from a multinomial logistic regression. The Trend column gives the linear dose-response association for the symptom across the numbers of GHQ caseness. The GHQ items are all scored on a 4-point response scale, with higher values indicating higher psychological distress. $\mathrm{N}=8,405$ person-observations of 5,954 participants with $\mathrm{GHQ} \geq 3$ at data-cycle baseline. 


\section{References}

1. Goldberg D, Goodyer I. The origins and course of common mental disorders. Routledge; 2005.

2. Rai D, Kosidou K, Lundberg M, Araya R, Lewis G, Magnusson C. Psychological distress and risk of long-term disability: Population-based longitudinal study. J Epidemiol Community Health. 2012 Jul;66(7):586-92.

3. Kivimäki M, Lawlor DA, Singh-Manoux A, Batty GD, Ferrie JE, Shipley MJ, et al. Common mental disorder and obesity-insight from four repeat measures over 19 years: Prospective Whitehall II cohort study. BMJ. 2009;339(7726).

4. Jokela M, Singh-Manoux A, Shipley MJ, Ferrie JE, Gimeno D, Akbaraly TN, et al. Natural course of recurrent psychological distress in adulthood. J Affect Disord. 2011;130(3):45461.

5. Wakefield JC, Schmitz MF. When does depression become a disorder? Using recurrence rates to evaluate the validity of proposed changes in major depression diagnostic thresholds. World Psychiatry. 2013;12(1):44-52.

6. Wakefield JC, Schmitz MF. Predictive validation of single-episode uncomplicated depression as a benign subtype of unipolar major depression. Acta Psychiatr Scand. 2014;129(6):445-57.

7. Jokela M, García-Velázquez R, Airaksinen J, Gluschkoff K, Kivimäki M, Rosenström T. Chronic diseases and social risk factors in relation to specific symptoms of depression: Evidence from the U.S. national health and nutrition examination surveys. J Affect Disord [Internet]. 2019 May;251:242-7. Available from: https://linkinghub.elsevier.com/retrieve/pii/S0165032718328453

8. García-Velázquez R, Jokela M, Rosenström TH. Symptom severity and disability in psychiatric disorders: The U.S. Collaborative Psychiatric Epidemiology Survey. J Affect Disord. 2017;222.

9. García-Velázquez R, Jokela M, Rosenström TH. The varying burden of depressive symptoms across adulthood: Results from six NHANES cohorts. J Affect Disord [Internet]. 2019 Mar;246:290-9. Available from: https://linkinghub.elsevier.com/retrieve/pii/S016503271831721X

10. Fried EI, Nesse RM. Depression sum-scores don't add up: why analyzing specific depression 
symptoms is essential. BMC Med [Internet]. 2015;13(1):72. Available from: http://bmcmedicine.biomedcentral.com/articles/10.1186/s12916-015-0325-4

11. Jokela M, Virtanen M, Batty GD, Kivimäki M. Inflammation and Specific Symptoms of Depression. JAMA Psychiatry [Internet]. 2015;73(1):1-6. Available from: http://archpsyc.jamanetwork.com/article.aspx?articleid=2470679

12. Wakefield JC, Schmitz MF. Severity of complicated versus uncomplicated subthreshold depression: New evidence on the "Monotonicity Thesis" from the national comorbidity survey. J Affect Disord. 2017;212:101-9.

13. Wakefield JC, Schmitz MF. Feelings of worthlessness during a single complicated major depressive episode predict postremission suicide attempt. Acta Psychiatr Scand. 2015;

14. GOLDBERG DP, GATER R, SARTORIUS N, USTUN TB, PICCINELLI M, GUREJE O, et al. The validity of two versions of the GHQ in the WHO study of mental illness in general health care. Psychol Med [Internet]. 1997 Jan 1;27(1):191-7. Available from: https://www.cambridge.org/core/product/identifier/S0033291796004242/type/journal_article

15. Buck N, McFall S. Understanding Society: design overview. Longit Life Course Stud. 2012 Jan 31;3(1).

16. Taylor MF, Brice J, Buck N, Prentice-lane E. British Household Panel Survey User Manual Volume a Introduction , Technical Report and Appendices. Inst Soc Econ Res. 2010;

17. Whaley CJ, Morrison DL, Payne RL, Fritschi L, Wall TD. Chronicity of Psychological Strain in Occupational Settings and the Accuracy of the General Health Questionnaire. J Occup Health Psychol [Internet]. 2005 Oct;10(4):310-9. Available from: http://doi.apa.org/getdoi.cfm?doi=10.1037/1076-8998.10.4.310

18. Richard C, Lamarche L, Lussier M-T, Gagnon R. GHQ-28 and cGHQ-28: implications of two scoring methods for the GHQ in a primary care setting. Soc Psychiatry Psychiatr Epidemiol [Internet]. 2004 Mar 1;39(3):235-43. Available from: http://link.springer.com/10.1007/s00127-004-0710-3

19. Goldberg DP, Williams P. A user's guide to the General Health Questionnaire. NFERNelson; 1988.

20. Fried EI. The 52 symptoms of major depression: Lack of content overlap among seven common depression scales. J Affect Disord [Internet]. 2017;208(October 2016):191-7. Available from: http://dx.doi.org/10.1016/j.jad.2016.10.019 
21. RUSCIO AM, LANE M, ROY-BYRNE P, STANG PE, STEIN DJ, WITTCHEN H-U, et al. Should excessive worry be required for a diagnosis of generalized anxiety disorder? Results from the US National Comorbidity Survey Replication. Psychol Med [Internet]. 2005 Dec 30;35(12):1761-72. Available from:

https://www.cambridge.org/core/product/identifier/S0033291705005908/type/journal_article

22. Wakefield JC, Schmitz MF. Uncomplicated depression is normal sadness, not depressive disorder: further evidence from the NESARC. World Psychiatry [Internet]. 2014 Oct;13(3):317-9. Available from: http://doi.wiley.com/10.1002/wps.20155 
Able to concentrate

One

Two

Three

Lost sleep over worry

One

Two

Three

Felt useful

One

Two

Three

Capable of decisions

One

Two

Three

Under strain

One

Two

Three

Overcoming difficulties

One

Two

Three

Enjoyed activities

One

Two

Three

Face up problems

One

Two

Three

Unhappy or depressed

One

Two

Three

Losing self-confidence

One

Two

Three

Worthlessness

One

Two

Three

Reasonably happy

One

Two

Three

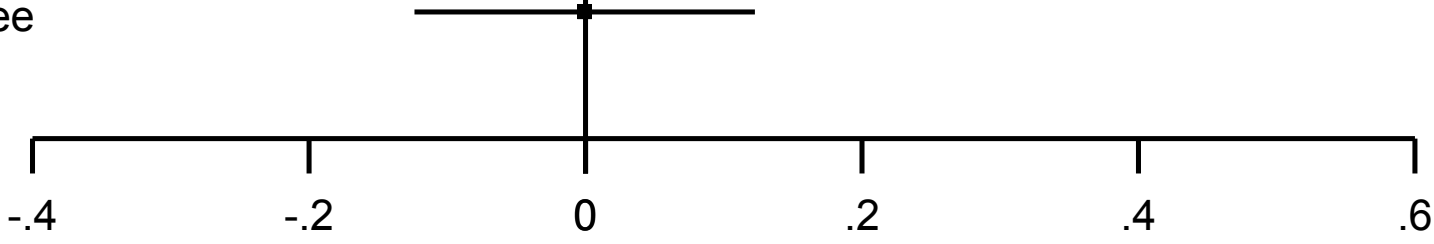

$\log R \mathrm{R}(95 \% \mathrm{Cl}) \quad$ Trend

$0.15(0.04,0.26)$

$0.06(-0.05,0.18)$

$0.22(0.10,0.35) \quad 0.04(-0.10,0.18)$

$0.03(-0.06,0.11)$

$0.16(0.07,0.25)$

$0.25(0.15,0.35) \quad 0.11(0.09,0.13)$

$-0.03(-0.13,0.06)$

$0.05(-0.05,0.15)$

$0.11(-0.00,0.22) \quad 0.07(0.06,0.08)$

$0.05(-0.07,0.16)$

$0.07(-0.05,0.19)$

$0.07(-0.06,0.20) \quad 0.01(0.00,0.02)$

$0.15(0.04,0.25)$

$0.19(0.08,0.31)$

$0.21(0.09,0.33) \quad 0.03(0.02,0.05)$

$0.09(-0.01,0.18)$

$0.12(0.01,0.22)$

$0.10(-0.01,0.21) \quad 0.01(-0.02,0.03)$

$0.05(-0.05,0.16)$

$0.17(0.05,0.28)$

$0.08(-0.05,0.20) \quad 0.01(-0.10,0.13)$

$0.03(-0.07,0.14)$

$0.07(-0.04,0.18)$

$0.20(0.08,0.33) \quad 0.09(0.03,0.14)$

$0.03(-0.08,0.14)$

$0.02(-0.10,0.14)$

$0.03(-0.10,0.16) \quad 0.00(-0.02,0.01)$

$0.16(0.06,0.25)$

$0.27(0.16,0.37)$

$0.30(0.18,0.41)$

$0.07(0.02,0.12)$

$0.09(0.01,0.18)$

$0.20(0.10,0.29)$

$0.45(0.36,0.55) \quad 0.18(0.09,0.27)$

$-0.13(-0.23,-0.02)$

$-0.07(-0.18,0.05)$

$-0.00(-0.12,0.12) \quad 0.06(0.06,0.07)$ 


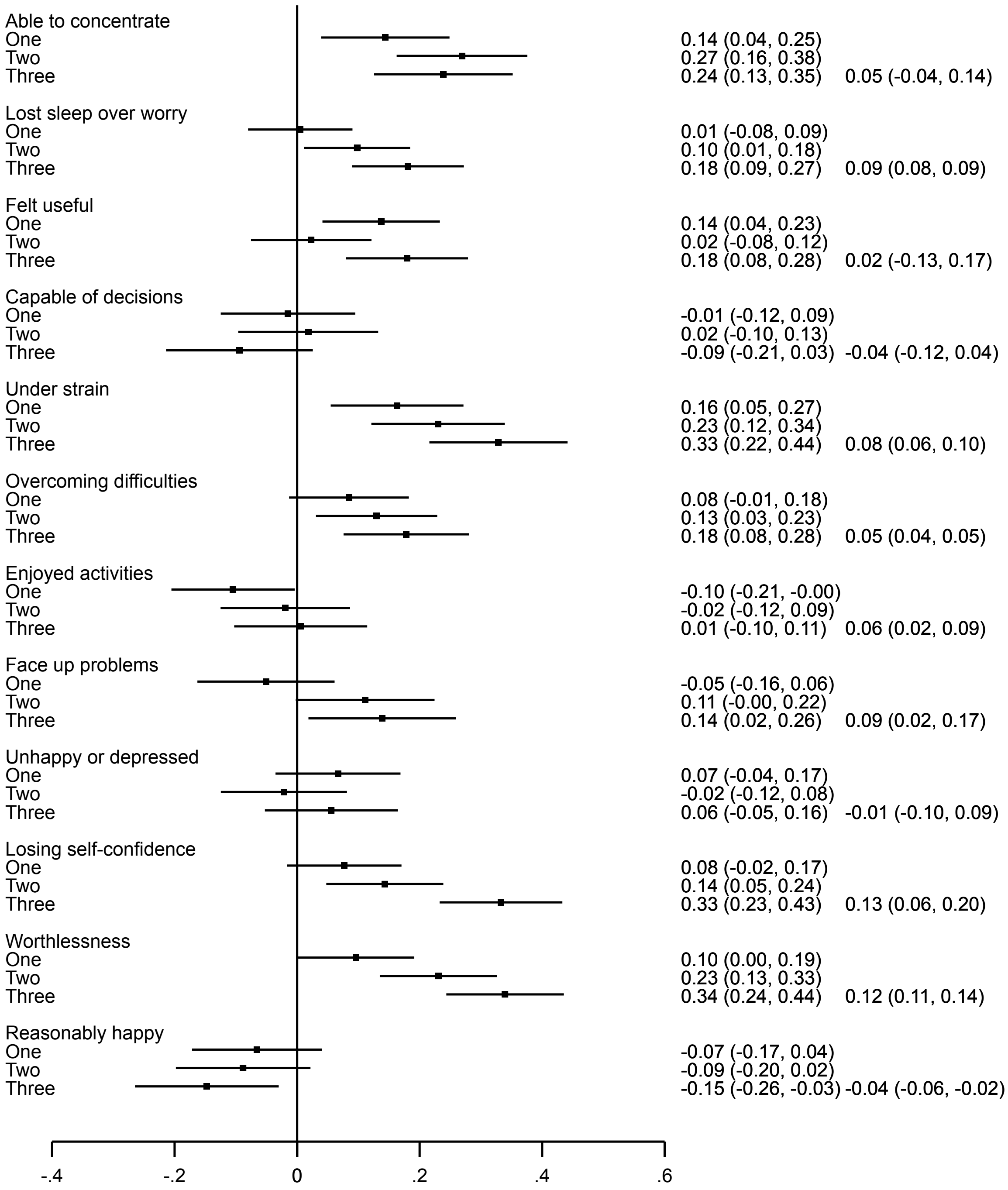


Specific symptoms of the General Health Questionnaire (GHQ) in predicting persistence of psychological distress: Data from two prospective cohort studies

Online Supplementary Material 


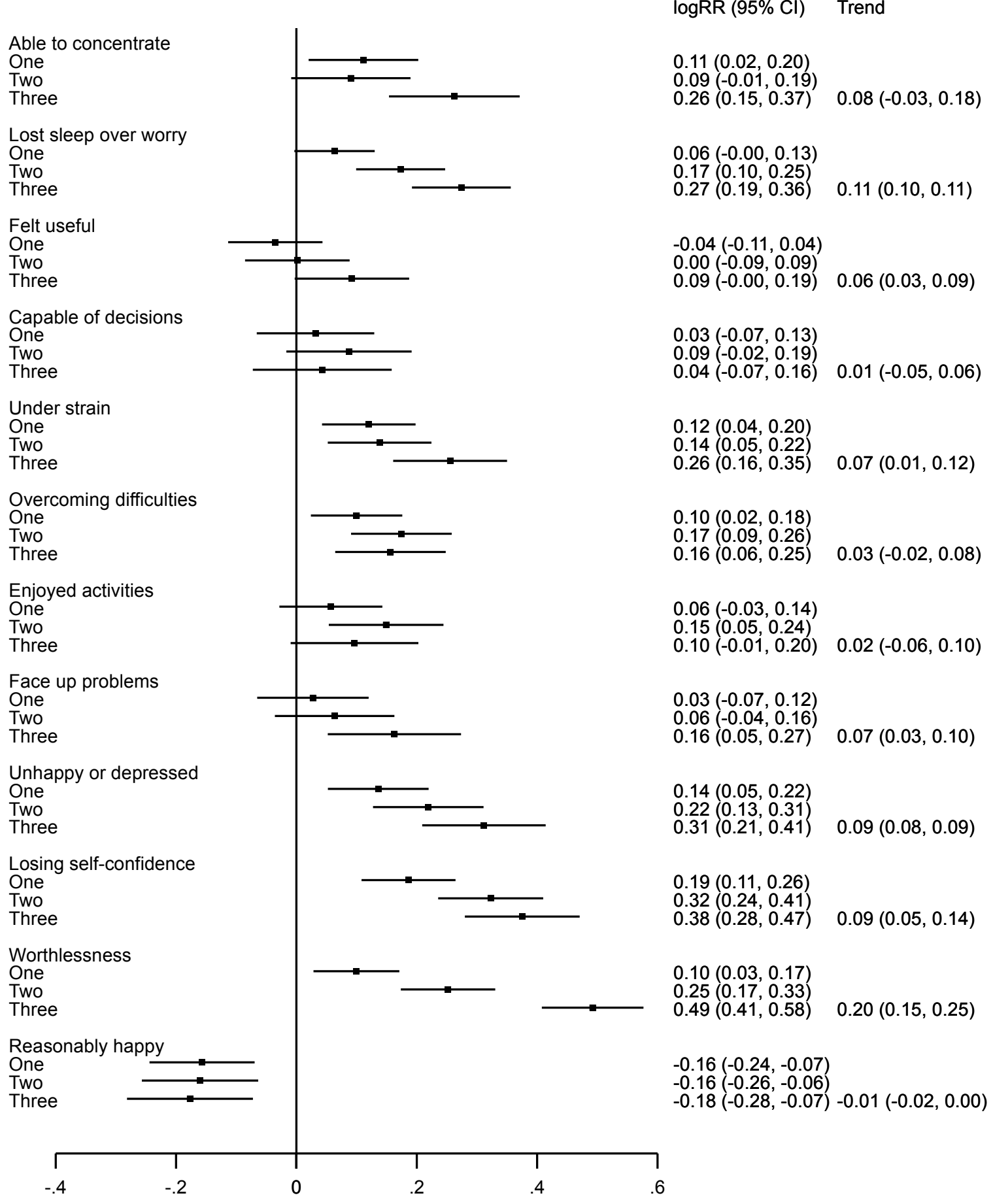

Supplementary Figure 1. Associations of GHQ-12 items in the imputed UKHLS data ( $\mathrm{n}=12,817$ person-observations of 10,816 participants with $\mathrm{GHQ} \geq 3$ at baseline) in predicting the number of times the participant was a GHQ case in the following 3 study waves (One, Two, Three; the reference group being zero). Values are natural logarithms of relative risks derived from a multinomial logistic regression. The Trend column gives the linear dose-response association for the symptom across the numbers of GHQ caseness. The GHQ items are all scored on a 4-point response scale, with higher values indicating higher psychological distress. 


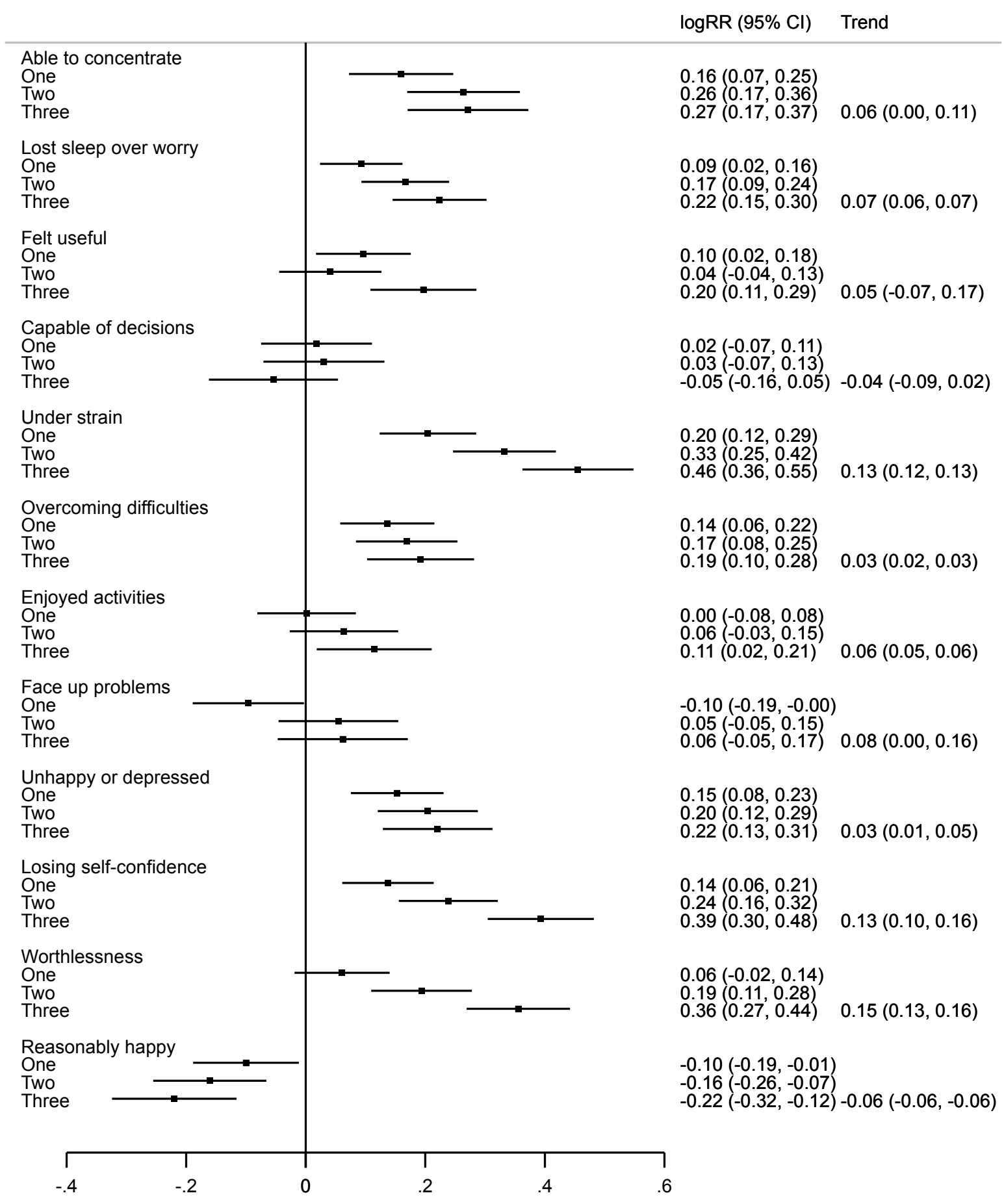

Supplementary Figure 2. Associations of GHQ-12 items in the imputed BHPS data ( $\mathrm{n}=19,879$ person-observations of 9,766 participants with GHQ $\geq 3$ at baseline) in predicting the number of times the participant was a GHQ case in the following 3 study waves (One, Two, Three; the reference group being zero). Values are natural logarithms of relative risks derived from a multinomial logistic regression. The Trend column gives the linear dose-response association for the symptom across the numbers of GHQ caseness. The GHQ items are all scored on a 4-point response scale, with higher values indicating higher psychological distress. 


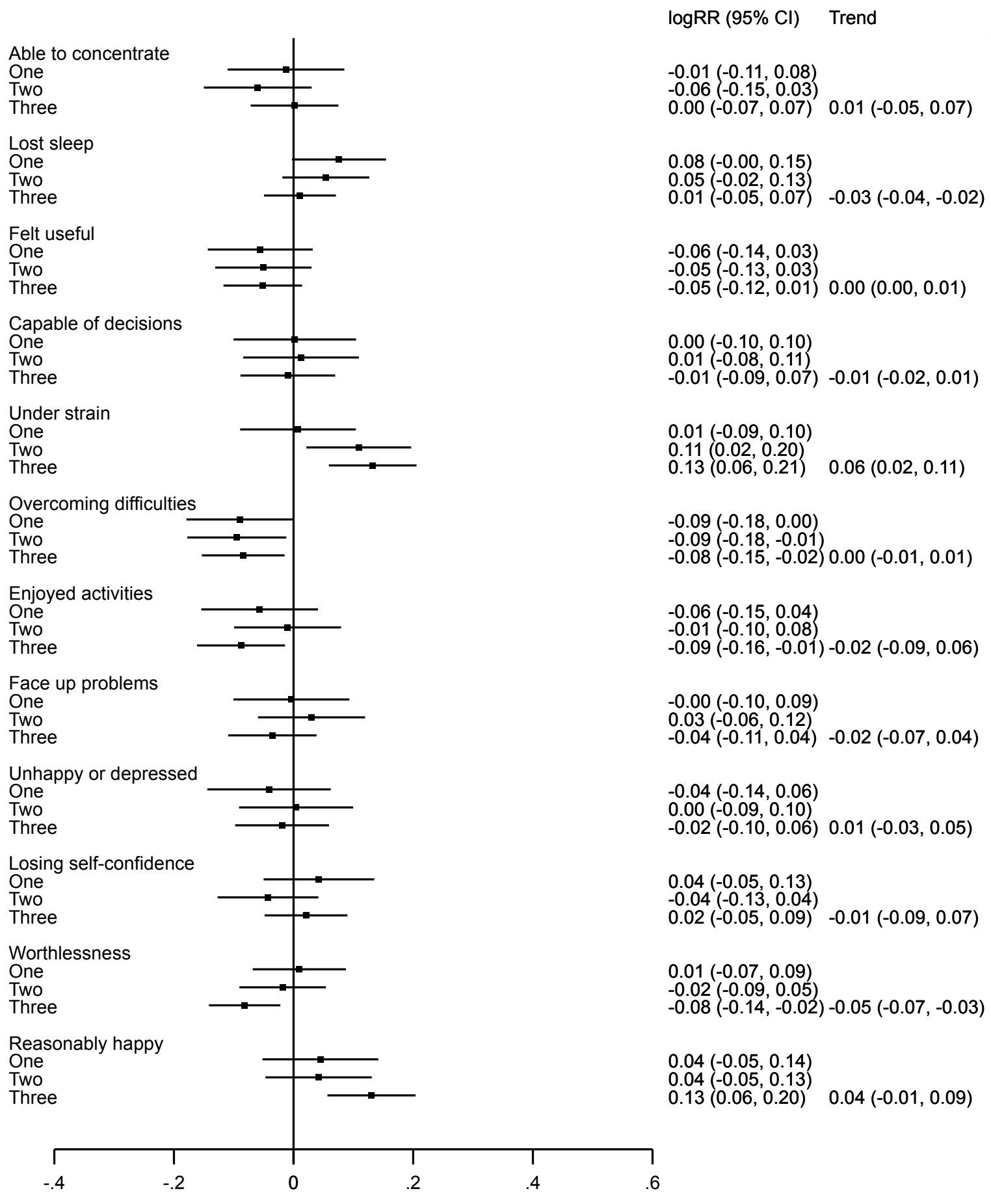

Supplementary Figure 3. Associations of GHQ-12 items in the UKHLS data ( $n=7,153$ personobservations of 6,430 participants with $\mathrm{GHQ} \geq 3$ at baseline) in predicting the number of times the participant was a Chronic GHQ case (CGHQ) in the following 3 study waves (One, Two, Three; the reference group being zero). Values are natural logarithms of relative risks derived from a multinomial logistic regression. The Trend column gives the linear dose-response association for the symptom across the numbers of GHQ caseness. The GHQ items are all scored on a 4-point response scale, with higher values indicating higher psychological distress. 


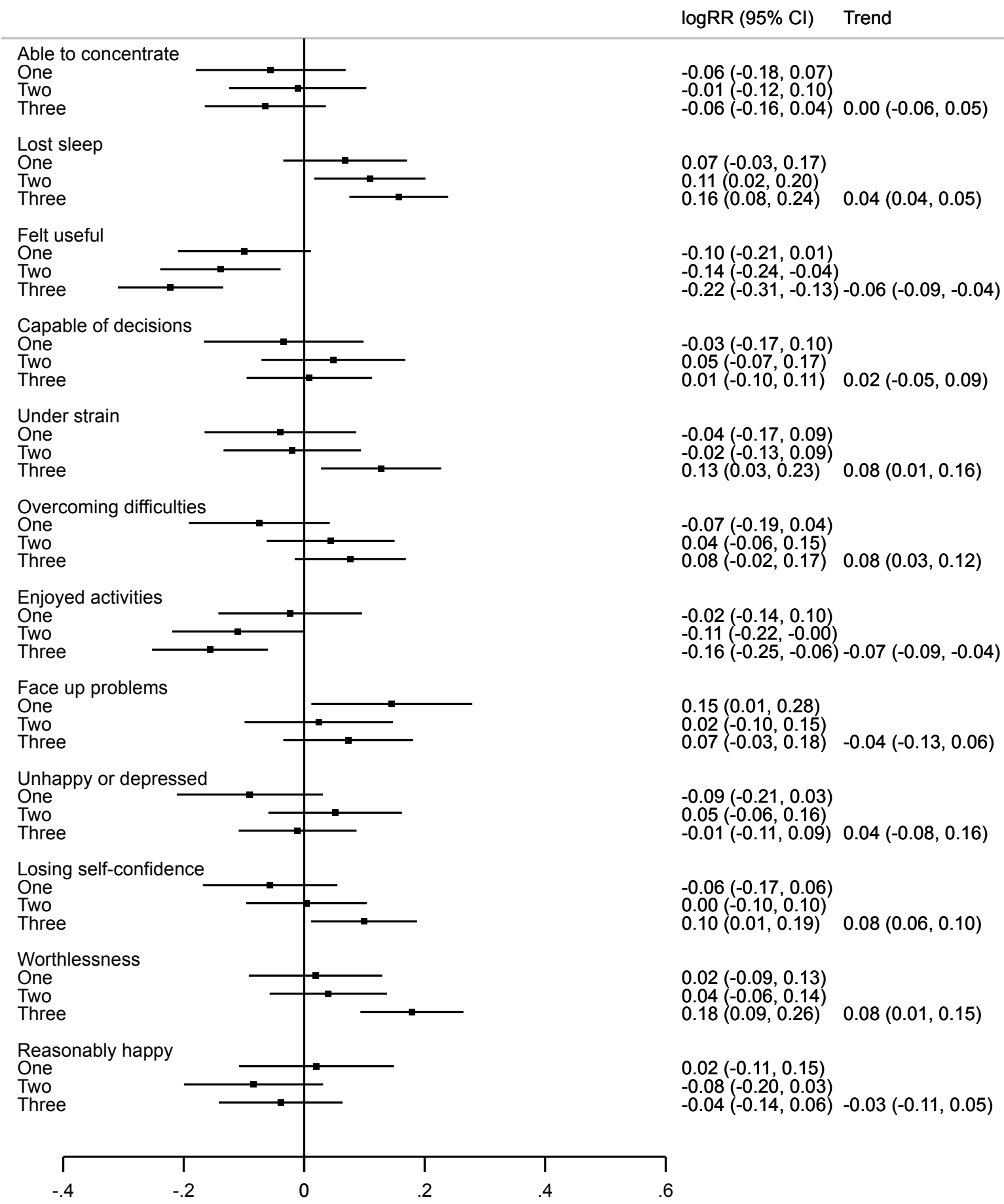

Supplementary Figure 4. Associations of GHQ-12 items in the BHPS data ( $n=8,405$ personobservations of 5,954 participants with $\mathrm{GHQ} \geq 3$ at baseline) in predicting the number of times the participant was a Chronic GHQ case (CGHQ) in the following 3 study waves (One, Two, Three; the reference group being zero). Values are natural logarithms of relative risks derived from a multinomial logistic regression. The Trend column gives the linear dose-response association for the symptom across the numbers of GHQ caseness. The GHQ items are all scored on a 4-point response scale, with higher values indicating higher psychological distress. 